\title{
'H MRS Study of Dorsolateral Prefrontal Cortex in Healthy Individuals before and after Lithium Administration
}

\author{
Paolo Brambilla ${ }^{1,2,3}$, Jeffrey A Stanley ${ }^{4}$, Roberto B Sassi ${ }^{4,5}$, Mark A Nicoletti', Alan G Mallinger ${ }^{4,6}$, \\ Matcheri S Keshavan ${ }^{4}$ and Jair C Soares*, ${ }^{*, 7,8}$ \\ 'Division of Mood and Anxiety Disorders, Department of Psychiatry, The University of Texas Health Science Center at San Antonio, San Antonio, \\ TX, USA; ${ }^{2}$ Section of Psychiatry, Department of Pathology and Experimental and Clinical Medicine, University of Udine School of Medicine, Udine, \\ Italy; ${ }^{3}$ Advanced Biotechnology Center, University of Genova, Genova, Italy; ${ }^{4}$ Department of Psychiatry, Western Psychiatric Institute and Clinic, \\ University of Pittsburgh School of Medicine, Pittsburgh, PA, USA; ${ }^{5}$ Department of Psychiatry, University of Sao Paulo School of Medicine, Brazil; \\ ${ }^{6}$ Department of Pharmacology, University of Pittsburgh School of Medicine, Pittsburgh, PA, USA; ${ }^{7}$ Department of Radiology, The University of \\ Texas Health Science Center at San Antonio, San Antonio, TX, USA; ${ }^{8}$ South Texas Veterans Health Care System, Audie L. Murphy Division, San \\ Antonio, TX, USA
}

\begin{abstract}
The mechanism of action of lithium is still largely unknown. However, recent animal and human studies suggested the possible neuroprotective effects of this medication. In particular, a recent magnetic resonance spectroscopy (MRS) study showed the increase of cortical brain levels of N-acetyl-aspartate (NAA), a putative marker of neuronal integrity/functioning, in both bipolar patients and normal controls after 4 weeks of lithium administration. We investigated the effects of lithium on NAA levels in a sample of healthy individuals using in vivo 'H MRS in dorsolateral prefrontal cortex (DLPFC), a region likely implicated in the pathophysiology of bipolar disorder. In vivo short echo-time 'H-MRS measurements of $8 \mathrm{~cm}^{3}$ single voxels placed bilaterally in the DLPFC were conducted at baseline and after 4 weeks of lithium administration on 12 healthy individuals (mean age $\pm S D=25.0 \pm 9.8$ years; six males). After lithium administration, no significant differences in NAA, phosphocreatine plus creatine, glycerophosphocholine plus phosphocholine (or choline-containing molecules), and myo-inositol absolute levels or ratios were found in DLPFC (paired t-tests, $p>0.05$ ). Contrary to prior MRS reports in bipolar patients, we found that lithium administration did not significantly increase NAA levels in the DLPFC of healthy individuals. Future longitudinal studies will be needed to further investigate whether chronic lithium treatment increases NAA levels in other brain regions in healthy individuals, and whether it promotes changes in these levels in specific brain regions in bipolar patients.

Neuropsychopharmacology (2004) 29, 1918-1924, advance online publication, I4 July 2004; doi: I0.1038/sj.npp. 1300520
\end{abstract}

Keywords: proton magnetic resonance spectroscopy; lithium; healthy individuals; glutamate; $N$-acetyl aspartate; prefrontal cortex

\section{INTRODUCTION}

Lithium is a first-line drug for acute and prophylactic treatment of bipolar disorder (Brambilla et al, 2003; Soares and Gershon, 1998). Its mechanism of action has not been elucidated, although several preclinical studies have tried to better clarify its biological basis (Lenox and Hahn, 2000). Specifically, in vitro and ex vivo studies reported that lithium facilitates long-term neurotrophic effects in rodent

\footnotetext{
*Correspondence: Dr JC Soares, Division of Mood and Anxiety Disorders, Department of Psychiatry (MC 7792), The University of Texas Health Science Center at San Antonio, 7703 Floyd Curl Drive, San Antonio, TX 78229, USA, Tel: + I 2105675492 , Fax: + I 210 567 3759, E-mail: soares@uthscsa.edu

Received 13 November 2003; revised 3 June 2004; accepted 3 June 2004

Online publication: 4 June 2004 at http://www.acnp.org/citations/ Npp06040303525/default.pdf
}

brain and in human neuronal cells, probably by increasing the levels of important cytoprotective proteins, such as bcl2 , and/or by decreasing the levels of specific proapoptotic proteins (eg p53, Bax) (Chen et al, 1999; Chen and Chuang, 1999; Chuang et al, 2002; Jope, 1999; Manji et al, 2000). ${ }^{1} \mathrm{H}$ magnetic resonance spectroscopy (MRS) represents a novel noninvasive method that can directly assess in vivo brain levels of specific chemicals of interest (Brambilla et al, 2002a; Stanley, 2002a), including $\mathrm{N}$-acetyl-aspartate (NAA), a brain metabolite thought to be a marker of neuronal viability and function (Pouwels and Frahm, 1998; Stanley et al, 2000). An MRS study reported increased cortical brain levels of NAA in a group of both bipolar and healthy subjects after 4 weeks of lithium treatment (Moore et al, 2000a). Additionally, increased total brain gray matter volumes in bipolar patients have been suggested by controlled MRI studies in patients who had been on lithium treatment for 4 (Moore et al, 2000b) and 27 (median) weeks 
(Sassi et al, 2002). Taken together, these findings suggest that lithium has neuroprotective effects in the human brain, which could be detectable in vivo with brain imaging.

However, it is still unclear whether any NAA changes induced by lithium are region-specific, and whether they would take place in healthy brains of normal individuals. Therefore, it is of great interest to examine the dorsolateral prefrontal cortex (DLPFC; ie, Brodmann's areas 9 and 46) of healthy individuals with ${ }^{1} \mathrm{H}$ MRS before and after lithium administration. The DLPFC was chosen as our target region as in recent years, structural, functional, and neurochemical brain imaging studies have implicated abnormalities in prefrontal cortex circuits in the pathophysiology of mood disorders (Brambilla et al, 2002b; Morice, 1990; Sax et al, 1999; Soares and Mann, 1997). The DLPFC is an important area of the prefrontal cortex receiving projections from higher order association centers and playing a key role in cognitive and executive brain functions (Nauta, 1971; Quintana and Fuster, 1999). Specifically, a role for the DLPFC in the pathophysiology of bipolar disorder is supported by recent MRS and postmortem studies showing reductions in NAA levels and in neuronal and glial density in DLPFC of bipolar patients (Rajkowska et al, 2001; Winsberg et al, 2000). Furthermore, DLPFC stimulation with transcranial magnetic stimulation (TMS) appeared to be effective in treating refractory depression in bipolar patients (George et al, 2000).

In the present study, in vivo ${ }^{1} \mathrm{H}$ MRS brain measures were performed in the DLPFC in a group of healthy individuals before and after 4 weeks of lithium administration. The main aim of this study was to investigate NAA changes in the DLPFC after lithium treatment in order to further test the hypothesis that it confers neuroprotection, which would be detectable in the in vivo human brain with ${ }^{1} \mathrm{H}$ MRS. Based on pre-clinical findings and prior human literature, we expected that healthy subjects would have a detectable elevation in NAA levels in the DLPFC after 4 weeks of lithium treatment.

\section{METHODS}

\section{Subjects}

In all, 12 healthy individuals with no DSM-IV axis I psychiatric disorders, as determined by the SCID-IV nonpatient version (SCID-NP), were studied. The subjects also had a full medical history and physical examination, including an electrocardiogram (ECG), blood tests to assess blood count, electrolytes, kidney, and liver functions, as well as urine test. Healthy subjects were excluded if there was use of any psychoactive medication within 2 weeks of the study, history of substance abuse/dependence, current medical problems, abnormalities in the blood, urine or ECG tests, or history of psychiatric disorders among self or first-degree relatives. All subjects were studied with ${ }^{1} \mathrm{H}$ MRS at baseline and after 4 weeks of lithium administration. The starting dose of lithium was $300 \mathrm{mg} /$ day, increased to $600 \mathrm{mg} /$ day at the second day of administration and maintained at such dosages for the first week. At the end of the first week, the 12-h lithium blood level was measured, and if it was below $0.6 \mathrm{mEq} / \mathrm{l}$, dosages were increased accordingly in order to reach the minimal therapeutic levels of $0.6 \mathrm{mEq} / \mathrm{l}$. All individuals provided signed informed consent, after having understood all issues involved in participation in the study protocol. This research study was approved by the University of Pittsburgh biomedical IRB.

\section{${ }^{1} \mathrm{H}$ MRI/MRS Procedure}

In vivo ${ }^{1} \mathrm{H}$ MRS measurements were conducted in a $1.5 \mathrm{~T}$ GE Signa Imaging System (General Electric Medical Systems, Milwaukee, WI). A set of sagittal and coronal scout images was first obtained to verify the patient position, image quality, positioning the voxel, and locate a midline sagittal image. In order to minimize the variance due to head position, a plastic head holder was used to allow placement of the subject's head in the same position, and a standardized procedure was utilized in order to obtain uniform head positioning and voxel placement during the pre- and post-lithium scans. A 3D spoiled gradient recalled (SPGR) acquisition was performed in the coronal plane $\left(\mathrm{TR}=25 \mathrm{~ms}, \mathrm{TE}=5 \mathrm{~ms}\right.$, flip angle $=40^{\circ}, \mathrm{FOV}=24 \mathrm{~cm}$, slice thickness $=1.5 \mathrm{~mm}, \mathrm{NEX}=1$, matrix size $=256 \times 192$ ) to obtain 124 images covering the entire brain. In all, 45-56 sagittal slices covering the entire brain were also obtained using a fast spin echo (FSE) sequence ( $T R=25 \mathrm{~ms}$, $\mathrm{TE}=17 \mathrm{~ms}$, flip angle $=40^{\circ}, \mathrm{FOV}=24 \mathrm{~cm}$, slice thickness $=3 \mathrm{~mm}, \mathrm{NEX}=1$, matrix size $=256 \times 192)$. The gray matter, white matter, and cerebrospinal fluid (CSF) content in the MRS voxels of interest were determined by performing segmentation with a semi-automated histogram method, which was applied to the SPGR data using the NIH Image software package, version 1.62 (National Institutes of Health, Bethesda, MD), as previously reported (Keshavan et al, 1994, 1995).

A double spin echo sequence was also used to obtain $T_{2}$ and proton density images in the axial plane to screen for neuroradiological abnormalities. A single-voxel short $\mathrm{TE}{ }^{1} \mathrm{H}$ spectrum of the right and left DLPFC were collected with a STEAM localization technique $(\mathrm{TE}=20 \mathrm{~ms}, \mathrm{TM}=13.6 \mathrm{~ms}$, $\mathrm{TR}=6 \mathrm{~s}$, bandwidth $=2 \mathrm{kHz}, 2048$ complex data points, 96 acquisitions, voxel dimension $2.0 \times 2.0 \times 2.0 \mathrm{~cm}^{3}$ ) (Figure 1). Additionally, water unsuppressed spectra were also collected for absolute quantification $(\mathrm{TR}=10 \mathrm{~s}$ and 16 acquisitions). The position of the voxel was visually inspected and adjusted based on identifiable anatomical landmarks in reference to standard brain atlases (Jackson and Duncan, 1996; Yuh et al, 1994). The superior frontal sulcus, the lateral fissure, and the genu of corpus callosum were used as anatomical boundaries for the voxel placement.

The post-processing and quantification steps for the short TE STEAM ${ }^{1} \mathrm{H}$ MRS data were $100 \%$ automated. The unsuppressed water spectrum was used to correct for any Eddy current effects. No apodization was applied and any residual water signal was removed by using the operatorindependent SVD-based method (de Beer et al, 1992). Five Gaussian damped sinusoids were used to model the in vivo data in the time domain using the Marquardt algorithm (NAA at $2.01 \mathrm{ppm}$, phosphocreatine + creatine $(\mathrm{PCr}+\mathrm{Cr})$ at $3.02,3.93 \mathrm{ppm}$, glycerophosphocholine plus phosphocholine (GPC + PC or the choline-containing metabolites) at $3.21 \mathrm{ppm}$ and myo-inositol (INO) at $3.54 \mathrm{ppm}$ ). To ensure 
that the signals of overlapping and of lesser amplitudes (ie metabolites with multiplet structures and macromolecules) have negligible influence on the fitting of the singlets, the first $37 \mathrm{~ms}$ of the free-induction decay (FID) signal were omitted in the fitting, which has been shown to reliably and accurately quantify NAA, PCr $+\mathrm{Cr}$, and GPC + PC (Stanley et al, 2002b) (Figure 2). INO levels were also quantified because of their relevance for the mechanism of action of lithium (Jope, 1999), although they have large variance and are less reliable to quantify with the utilized method. The
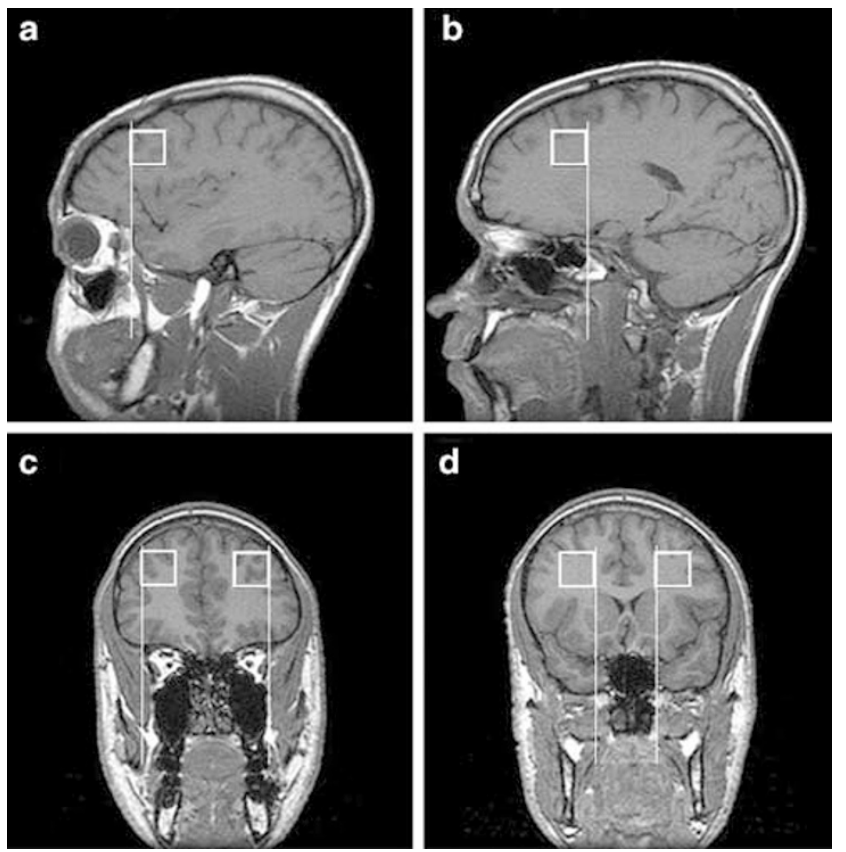

Figure I The white box represents the location of the volume of interest $(2 \times 2 \times 2 \mathrm{cc})$ in the left and right DLPFC. A single-voxel short TE ' $\mathrm{H}$ spectrum was collected with a STEAM localization technique ( $T E=20 \mathrm{~ms}, T M=13.6 \mathrm{~ms}$, TR=6s). The thin lines in pictures (a) and (b) represent the position of the coronal images in (c) and (d). The thin lines in (c) and (d) represent the position of the sagittal images in (a) and (b). The superior frontal sulcus, the lateral fissure, and the genu of corpus callosum were used as anatomical boundaries for the voxel placement. unsuppressed water signal, gray matter, white matter and CSF voxel content values, along with the other appropriate correction factors, were utilized to obtain absolute quantification values with units of $\mathrm{mmol} / \mathrm{kg}$ wet weight, as previously described by Stanley et al (1995).

\section{Statistical Analyses}

All analyses were performed using the SPSS for Windows software, version 11.1 (SPSS Inc., Chicago), and two-tailed significance level was set at $p<0.05$. Paired $t$-tests were performed to determine whether the measured ${ }^{1} \mathrm{H}$ metabolite levels and ratios differed between pre- and post-lithium administration. The relationship between changes in measured metabolite levels after lithium administration and lithium blood levels were examined using Pearson's correlation coefficient.

\section{RESULTS}

Healthy subjects had a mean age of 25.0 years with an SD of 9.8 (range: $18-52$ years). Six of them were males. The mean and SD for the $12 \mathrm{~h}$ lithium blood levels at the end of the 4 th week were 0.83 and $0.20 \mathrm{mEq} / \mathrm{l}$, respectively, with a mean lithium dosage of $1262.50 \mathrm{mg} /$ day and an SD of 216.50 .

No significant differences for gray matter, white matter, and CSF voxel contents were found before and after lithium administration (paired $t$-tests, $p>0.05$ ) (Table 1). Absolute metabolite levels for NAA, PCr $+\mathrm{Cr}$, GPC $+\mathrm{PC}$, INO, and ratios did not differ significantly in left or right DLPFC after lithium treatment (Paired $t$-tests, $p>0.05$ ) (Table 2; Figure 3$)$. The coefficients of variation (CV), expressing the standard deviation as a percentage of the sample mean of the combined pre- and post-lithium measurements, ranged from $7.5 \%(\mathrm{PCr}+\mathrm{Cr})$ to $24.6 \%(\mathrm{GPC}+\mathrm{PC})$ in the left DLPFC and from $11.5 \%$ (NAA) to $34.1 \%(\mathrm{GPC}+\mathrm{PC} /$ $\mathrm{PCr}+\mathrm{Cr}$ ) for the right DLPFC (Table 2). The mean percentage change of metabolite levels (ie post-lithium measurement minus pre-lithium measurement divided by the pre-lithium measurement) ranged from $7.2 \%(\mathrm{PCr}+\mathrm{Cr})$ to $25.0 \%$ (INO) for the left side and from $11.5 \%$ (NAA) to

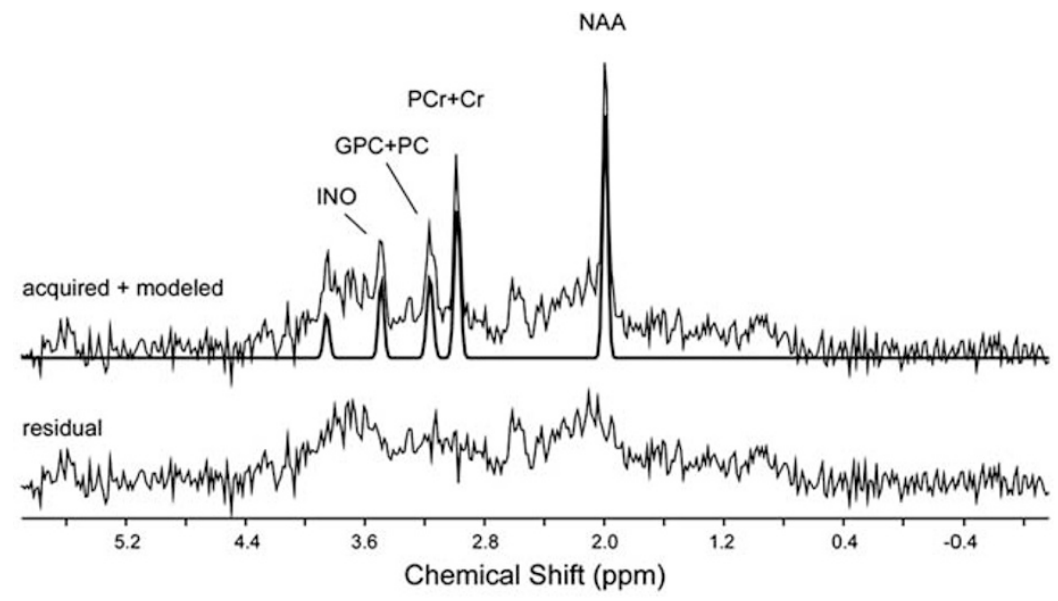

Figure 2 A representative in vivo short TE spectrum of the DLPFC showing the Fourier transform of the acquired signal (thin line) and the modeled signal (thick line) with the residual below. The quantified singlets, NAA, PCr $+\mathrm{Cr}, \mathrm{GPC}+\mathrm{PC}$, and INO are labeled accordingly. 
$43.5 \%$ (INO) for the right side, and were all lower or comparable to the respective coefficients of variation, except for INO levels in right DLPFC (Table 2).

No significant correlation between changes in absolute levels of NAA, PCr $+\mathrm{Cr}$, GPC + PC, and INO after lithium administration and lithium blood levels were found (Pearson's correlation coefficient, $p>0.05$ ).

Table I 'H MRS Voxel Content in Dorsolateral Prefrontal Cortex in 12 Healthy Subjects

\begin{tabular}{|c|c|c|c|c|c|c|}
\hline & \multicolumn{2}{|c|}{ Baseline } & \multicolumn{2}{|c|}{ Post lithium } & \multicolumn{2}{|c|}{ Paired t-test } \\
\hline & Mean & SD & Mean & SD & $t(d f=I I)$ & $p$ \\
\hline \multicolumn{7}{|l|}{ Left DLPFC } \\
\hline Gray matter & 0.476 & 0.096 & 0.517 & 0.089 & -1.158 & 0.271 \\
\hline White matter & 0.524 & 0.096 & 0.483 & 0.089 & 1.158 & 0.271 \\
\hline CSF & 0.003 & 0.003 & 0.002 & 0.003 & 1.148 & 0.275 \\
\hline
\end{tabular}

\section{Right DLPFC}

$\begin{array}{lllllrl}\text { Gray matter } & 0.422 & 0.110 & 0.484 & 0.079 & -2.003 & 0.070 \\ \text { White matter } & 0.578 & 0.110 & 0.516 & 0.079 & 2.003 & 0.070 \\ \text { CSF } & 0.003 & 0.003 & 0.001 & 0.002 & 1.126 & 0.283\end{array}$

DLPFC $=$ dorsolateral prefrontal cortex; $\mathrm{df}=$ degrees of freedom. The gray matter, white matter, and CSF measures are $\%$ values.

\section{DISCUSSION}

Our present study did not find significant changes in NAA levels in DLPFC of healthy individuals after 4 weeks of lithium administration. This study did not confirm the possible role of lithium in modulating cortical NAA levels in healthy volunteers. However, lithium's neuroprotective properties may require longer administration at therapeutic concentrations, despite the fact that it is well known from clinical studies that lithium's therapeutic effects in bipolar patients are already detectable after 3-4 weeks of treatment.

Contrary to our findings, Moore et al (2000a) reported a small but significant increase in total brain NAA levels (5\%, using non-normalized NAA concentrations) after 4 weeks of lithium treatment in 21 individuals, including 12 bipolar patients and nine healthy subjects. This was suggested by the authors to be putatively related to the reported increased levels of neurotrophic brain factors, such as bcl-2, after chronic lithium treatment (Manji et al, 2000). However, in the study by Moore et al (2000a), therapeutic plasma levels of lithium $(0.8 \mathrm{mEq} / \mathrm{l})$ were reached over the first week of treatment, whereas in our study it took longer to reach therapeutic levels in several individuals. Specifically, in our study, at the third week of treatment lithium blood levels were below $0.6 \mathrm{mEq} / \mathrm{L}$ in six subjects. Thus, by using a more aggressive lithium titration schedule, Moore et al (2000a) may have seen faster effects of lithium in increasing NAA levels, already detectable at week 4, compared to our investigation. Furthermore, Moore et al (2000a) investigated lithium effects in a mixed sample of

Table 2 'H MRS Measures in Dorsolateral Prefrontal Cortex in 12 Healthy Subjects at Baseline and After 4 Weeks of Lithium Administration

\begin{tabular}{|c|c|c|c|c|c|c|c|c|c|}
\hline & \multicolumn{2}{|c|}{ Baseline } & \multicolumn{2}{|c|}{ Post lithium } & \multicolumn{2}{|c|}{ Paired $t$-test } & \multirow[b]{2}{*}{ CV (\%) } & \multicolumn{2}{|c|}{$\%$ Change } \\
\hline & Mean & SD & Mean & SD & $t$ & $p$ & & Mean & SD \\
\hline \multicolumn{10}{|l|}{ Left DLPFC } \\
\hline NAA & 6.657 & 0.546 & 7.039 & 0.834 & -1.603 & 0.137 & 10.472 & 9.897 & 9.983 \\
\hline $\mathrm{PCr}+\mathrm{Cr}$ & 5.366 & 0.390 & 5.569 & 0.417 & -1.638 & 0.129 & 7.479 & 7.154 & 5.075 \\
\hline $\mathrm{NAA} / \mathrm{PC}+\mathrm{Cr}$ & 1.312 & 0.146 & 1.333 & 0.158 & -0.500 & 0.626 & 11.339 & 8.175 & 8.752 \\
\hline NAA/GPC+PC & 2.488 & 0.750 & 2.237 & 0.426 & 1.259 & 0.233 & 25.843 & 13.852 & 14.115 \\
\hline $\mathrm{GPC}+\mathrm{PC} / \mathrm{PCr}+\mathrm{Cr}$ & 0.563 & 0.151 & 0.615 & 0.131 & -1.346 & 0.205 & 24.019 & 21.522 & 31.418 \\
\hline \multicolumn{10}{|l|}{ Right DLPFC } \\
\hline INO & 3.533 & 1.229 & 3.197 & 0.638 & 0.790 & 0.445 & 28.922 & 43.542 & 36.000 \\
\hline $\mathrm{NAA} / \mathrm{PC}+\mathrm{Cr}$ & 1.273 & 0.178 & 1.356 & 0.129 & -1.192 & 0.258 & $12.04 \mid$ & 13.203 & 17.824 \\
\hline NAA/GPC+PC & 2.355 & 0.914 & 2.392 & 0.600 & -0.104 & 0.918 & 31.334 & 36.656 & 28.593 \\
\hline $\mathrm{GPC}+\mathrm{PC} / \mathrm{PC}+\mathrm{Cr}$ & 0.601 & 0.187 & 0.567 & 0.218 & 0.402 & 0.695 & 34.129 & 29.586 & 64.210 \\
\hline
\end{tabular}

$\mathrm{NAA}=\mathrm{N}$-acetyl-aspartate, $\mathrm{PCr}+\mathrm{Cr}=$ phosphocreatine plus creatine, $\mathrm{GPC}+\mathrm{PC}=$ choline-containing molecules, INO $=$ myo-inositol; $t=$ paired $t$-test, $p=$ significance; $\%$ change = percent change of variation relative to first measurement (difference between post- and pre-lithium conditions, divided by the pre-lithium condition); \% $\mathrm{CV}=$ coefficients of variation (defined as the standard deviation of the measurements divided by the mean) of the combined pre- and post-lithium measurements. Individual metabolite values are expressed as $\mathrm{mmol} / \mathrm{kg}$ wet weight. 
a

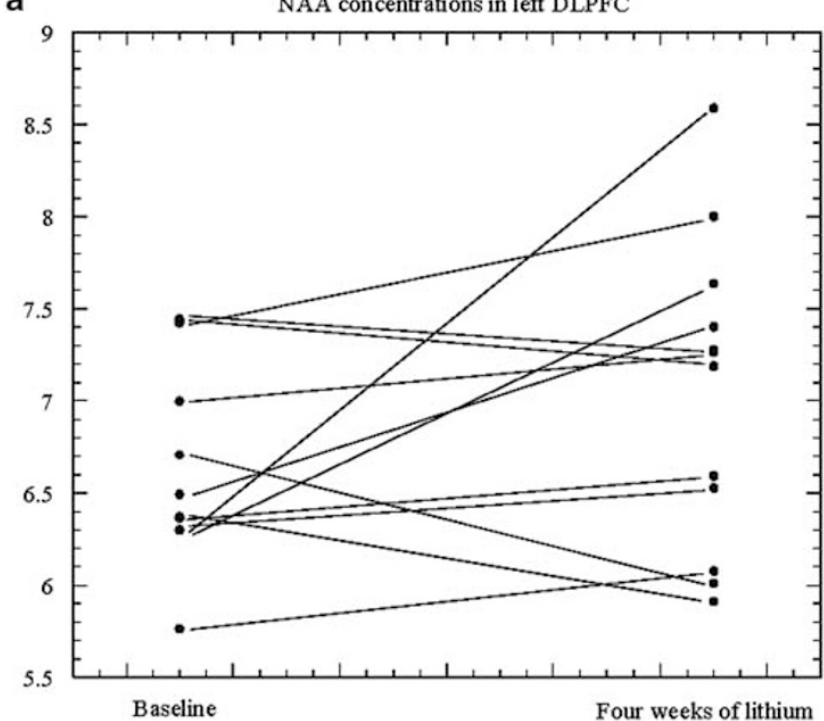

b

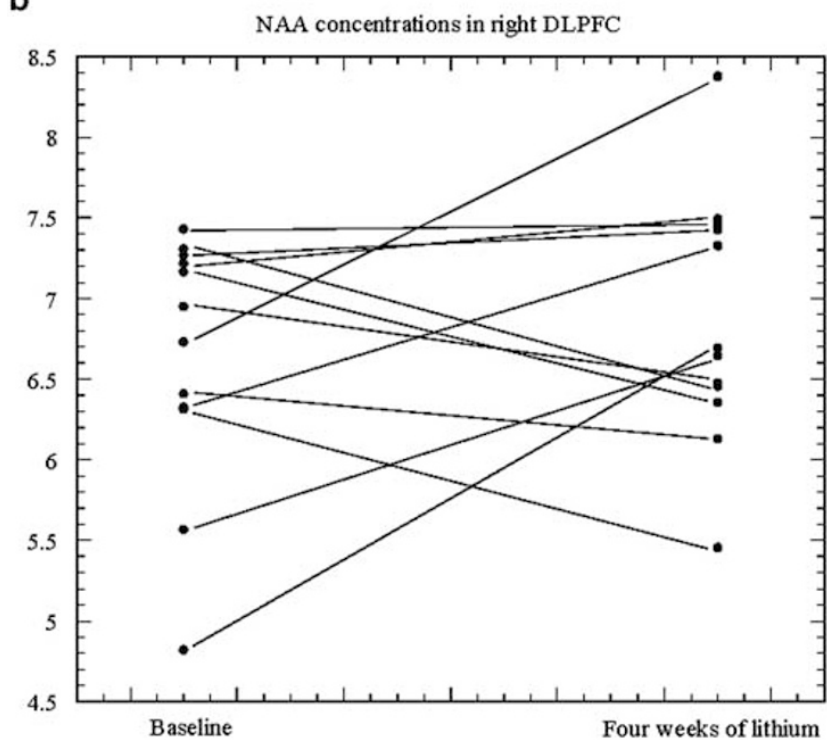

Figure 3 After 4 weeks of lithium administration in a sample of 12 healthy individuals, no significant changes in NAA concentrations were found in the left $(t=-1.603, d f=11, p=0.137)$ (Figure 2a) or right $\operatorname{DLPFC}(t=-0.832, d f=1 \mathrm{I}, p=0.422)$ (Figure $2 b)$.

depressed bipolar patients and healthy subjects. This was done to increase the statistical power of the study, according to the authors, as no significant differences in baseline NAA levels were detected between the two groups. Nonetheless, this limited the possibility of examining NAA changes in the two samples separately. On the contrary, in our study only healthy controls were examined, allowing the in vivo investigation of possible neurotrophic effects of lithium in the healthy human brain. It may be argued that the effects of lithium on NAA levels in healthy controls may be different compared to bipolar patients, as neuronal function in the prefrontal cortex of healthy individuals is unaffected. Moore et al (2000a) also examined different brain regions and combined NAA concentrations from four different voxel placements, that is, right frontal, left temporal, central occipital, and left parietal lobes, resulting in an overall increase in NAA of about $5 \%$ after lithium administration. Specifically, regional NAA levels increased by about $2.9 \%$ in left parietal lobe, $3.5 \%$ in right frontal lobe, $4.9 \%$ in left temporal lobe, and $7.3 \%$ in central occipital lobe. Thus, the discrepancy between our findings and those by Moore et al (2000a, b) may reflect region specificity, due to noncomparable regions of interest being utilized.

It should also be kept in mind that physiological systems may not respond to pharmacological interventions in healthy individuals the same way dysregulated systems do in patients with psychiatric illnesses (Heuser et al, 1996; Zanardi et al, 1997). So, it is possible that neuroprotective effects of lithium could be detected in brains of bipolar patients, where NAA deficits may be present (Renshaw et al, 1995; Winsberg et al, 2000), and possibly prevented or reversed by lithium treatment (Deicken et al, 2001; Silverstone et al, 2003; Sharma et al, 1992), but that no such effects would be present in healthy brain tissue of control individuals.

Consistent with prior MRS studies from Silverstone et al (1996, 1999a, b), who investigated the effects of 7 days of lithium administration in healthy volunteers, no significant changes in $\mathrm{NAA}, \mathrm{PCr}+\mathrm{Cr}$ and $\mathrm{GPC}+\mathrm{PC}$ levels were reported in our study in healthy individuals after 4 weeks of lithium treatment.

Some potential limitations of the present study should be taken in consideration. First, the relatively small sample size may have limited our ability to detect small changes in metabolite concentrations after lithium administration. Nonetheless, as suggested by the reported effect sizes from studies that examined possible medication changes on brain ${ }^{1} \mathrm{H}$ MRS metabolites (Brambilla et al, 2002a, b; Deicken et al, 1992; Lyoo et al, 2003), our study with 12 subjects had adequate statistical power. Second, only the DLPFC was investigated in this study. Therefore, even though there are no conclusive data to demonstrate that lithium's actions on ${ }^{1} \mathrm{H}$ MRS brain metabolites are region-specific, we can only comment with certainty on this particular brain region, and caution should be utilized when findings are extrapolated to other cortical brain areas. Last, the CV for GPC + PC was relatively high, although our method for MRS quantitation reliably and accurately resolves this metabolite along with $\mathrm{NAA}$ and $\mathrm{PCr}+\mathrm{Cr}$ (Stanley et al, 2002b). This may be due to the composite MRS signal reflecting GPC + PC, which includes several choline-containing molecules, primarily GPC and PC, but also free choline and acetylcholine (Miller, 1991).

In conclusion, this ${ }^{1} \mathrm{H}$ MRS study did not show increases of NAA levels in DLPFC after 4 weeks of lithium administration in healthy individuals. This is in contrast with a prior MRS study reporting significant increase in brain levels of NAA in both healthy subjects and bipolar patients, which would be consistent with detectable in vivo neuroprotective effects. To our best knowledge, this is the first in vivo MRS study investigating NAA levels in the DLPFC of healthy individuals before and after lithium administration. Future longitudinal studies will be needed to investigate whether chronic lithium treatment over longer periods of time may protect brain neurons and result in increase in NAA levels in DLPFC and other brain 
regions thought to be involved in the pathophysiology of mood disorders.

\section{ACKNOWLEDGEMENTS}

This study was partly supported by grants $\mathrm{MH} 01736$ and MH 30915 from the National Institute of Mental Health, NARSAD, the Krus Endowed Chair in Psychiatry (UTHSCSA), and the Veterans Administration. We thank Dick Drost (University of Western Ontario, London, Ontario, Canada) for assistance in providing the fitting software.

\section{REFERENCES}

Brambilla P, Barale F, Caverzasi E, Soares JC (2002b). Anatomical MRI findings in mood and anxiety disorders. Epidemiol Psychiatr Soc 11: 88-99.

Brambilla P, Barale F, Soares JC (2003). Atypical antipsychotics and mood stabilization in bipolar disorder. Psychopharmacology 166: 315-332.

Brambilla P, Stanley JA, Nicoletti M, Harenski K, Forster Wells K, Mallinger AG et al (2002a). H MRS brain measures and acute lorazepam administration in healthy human subjects. Neuropsychopharmacology 26: 546-551.

Chen G, Zeng WZ, Yuan PX, Huang LD, Jiang YM, Zhao ZH et al (1999). The mood-stabilizing agents lithium and valproate robustly increase the levels of the neuroprotective protein bcl2 in the CNS. J Neurochem 72: 879-882.

Chen RW, Chuang DM (1999). Long term lithium treatment suppresses p53 and Bax expression but increases Bcl-2 expression. A prominent role in neuroprotection against excitotoxicity. J Biol Chem 274: 6039-6042.

Chuang D-M, Chen R-W, Chaleka-Franaszek E, Ren M, Hashimoto $\mathrm{R}$, Senatorov $\mathrm{V}$ et al (2002). Neuroprotective effects of lithium in cultured cells and animal models of diseases. Bipolar Disord 4: 129-136.

de Beer R, van den Boogaart A, van Ormondt D, Pijnappel WWF, den Hollander JA, Marien AJH et al (1992). Application of timedomain fitting in the quantification of in vivo ${ }^{1} \mathrm{H}$ spectroscopic imaging data sets. NMR Biomed 5: 171-178.

Deicken RF, Calabrese G, Raz J, Sappey-Marinier D, Meyerhoff D, Dillon WP et al (1992). A 31phosphorous magnetic resonance spectroscopy study of diazepam does not affect brain phosphorous metabolism. Biol Psychiatry 32: 628-631.

Deicken RF, Eliaz Y, Feiwell R, Schuff N (2001). Increased thalamic $\mathrm{N}$-acetylaspartate in male patients with familial bipolar I disorder. Psychiatry Res: Neuroimaging 106: 35-45.

George MS, Nahas Z, Molloy M, Speer AM, Oliver NC, Li X et al (2000). A controlled trial of daily left prefrontal cortex TMS for treating depression. Biol Psychiatry 48: 962-970.

Heuser IJ, Schweiger U, Gotthardt U, Schmider J, Lammers CH, Dettling $\mathrm{M}$ et al (1996). Pituitary-adrenal-system regulation and psychopathology during amitriptyline treatment in elderly depressed patients and normal comparison subjects. Am J Psychiatry 153: 93-99.

Jackson GD, Duncan JS (1996). MRI Anatomy: A New Angle on the Brain. Churchill Livingstone: New York.

Jope RS (1999). Anti-bipolar therapy: mechanism of action of lithium. Mol Psychiatry 4: 117-128.

Keshavan MS, Anderson S, Beckwith C, Nash K, Pettegrew JW, Krishnan KR (1995). A comparison of stereology and segmentation techniques for volumetric measurements of lateral ventricles in magnetic resonance imaging. Psychiatry Res 61: 53-60.

Keshavan MS, Beckwith W, Pettegrew W, Krishnan KR (1994). An objective method for edge detection in MRI morphometry. Eur Psychiatry 9: 205-207.
Lenox RH, Hahn C-G (2000). Overview of the mechanism of action of lithium in the brain: fifty-year update. J Clin Psychiatry 61: 5-15.

Lyoo IK, Kong SW, Sung SM, Hirashima F, Parow A, Hennen J et al (2003). Multinuclear magnetic resonance spectroscopy of highenergy phosphate metabolites in human brain following oral supplementation of creatine-monohydrate. Psychiatry Res 123: 87-100.

Manji HK, Moore GJ, Chen G (2000). Lithium up-regulates the cytoprotective protein $\mathrm{Bcl}-2$ in the $\mathrm{CNS}$ in vivo: a role for neurotrophic and neuroprotective effects in maniac depressive illness. J Clin Psychiatry 61: 82-96.

Miller BL (1991). A review of chemical issues in ${ }^{1} \mathrm{H}$ NMR spectroscopy: $N$-acetyl-L-aspartate, creating and choline. $N M R$ Biomed 4: 47-52.

Moore GJ, Bebchuk JM, Hasanat K, Chen G, Seraji-Bozorgzad N, Wilds IB et al (2000a). Lithium increases $N$-acetyl-aspartate in the human brain: in vivo evidence in support of bcl-2's neurotrophic effects? Biol Psychiatry 48: 1-8.

Moore GJ, Bebchuck JM, Wilds IB, Chen G, Manji HK (2000b). Lithium-induced increase in human brain gray matter. Lancet 356: $1241-1242$.

Morice R (1990). Cognitive inflexibility and pre-frontal dysfunction in schizophrenia and mania. Br J Psychiatry 157: 50-54.

Nauta WJ (1971). The problem of the frontal lobe: a reinterpretation. J Psychiatr Res 8: 167-187.

Pouwels PJ, Frahm J (1998). Regional metabolite concentrations in human brain as determined by quantitative localized proton MRS. Magn Reson Med 39: 53-60.

Quintana J, Fuster JM (1999). From perception to action: temporal integrative functions of prefrontal and parietal neurons. Cerebral Cortex 9: 213-221.

Rajkowska G, Halaris A, Selemon LD (2001). Reductions in neuronal and glial density characterize the dorsolateral prefrontal cortex in bipolar disorder. Biol Psychiatry 49: 741-752.

Renshaw PF, Yurgelun-Todd DA, Tohen M, Gruber S, Cohen BM (1995). Temporal lobe proton magnetic resonance spectroscopy of patients with first-episode psychosis. Am J Psychiatry 152: 444-446.

Sassi RB, Nicoletti M, Brambilla P, Mallinger AG, Fran E, Kupfer DJ et al (2002). Increased gray matter volume in lithium-treated bipolar disorder patients. Neurosci Lett 329: 243-245.

Sax KW, Strakowski SM, Zimmerman ME, DelBello MP, Keck Jr PE, Hawkins JM (1999). Frontosubcortical neuroanatomy and the continuous performance test in mania. Am J Psychiatry 156: $139-141$.

Sharma R, Venkatasubramanian PN, Barany M, Davis JM (1992). Proton magnetic resonance spectroscopy of the brain in schizophrenic and affective patients. Schizophren Res 8: 43-49.

Silverstone PH, Hanstock CC, Fabian J, Staab R, Allen PS (1996). Chronic lithium does not alter human myo-inositol or phosphomonoester concentrations as measured by ${ }^{1} \mathrm{H}$ and ${ }^{31} \mathrm{P}$ MRS. Biol Psychiatry 40: 235-246.

Silverstone PH, Hanstock CC, Rotzinger S (1999a). Lithium does not alter the choline/creatine ratio in the temporal lobe of human volunteers as measured by proton magnetic resonance spectroscopy. J Psychiatry Neurosci 24: 222-226.

Silverstone PH, Rotzinger S, Pukhovsky A, Hanstock CC (1999b). Effects of lithium and amphetamine on inositol metabolism in the human brain as measured by ${ }^{1} \mathrm{H}$ and ${ }^{31} \mathrm{P}$ MRS. Biol Psychiatry 46: 1634-1641.

Silverstone $\mathrm{PH}, \mathrm{Wu} \mathrm{RH}$, O'Donnell T, Ulrich M, Asghar SJ, Hanstock CC (2003). Chronic treatment with lithium, but not sodium valproate, increases cortical $\mathrm{N}$-acetyl-aspartate concentrations in euthymic bipolar patients. Int Clin Psychopharmacolgy 18: 73-79.

Soares JC, Gershon S (1998). The lithium ion: a foundation for psychopharmacological specificity. Neuropsychopharmacology 19: $167-182$. 
Soares JC, Mann JJ (1997). The functional neuroanatomy of mood disorders. J Psychiatr Res 31: 393-432.

Stanley JA (2002a). In vivo magnetic resonance spectroscopy and its application to neuropsychiatric disorders. Canad J Psychiatry 47: $315-326$.

Stanley JA, Drost DJ, Williamson PC, Thompson RT (1995). The use of a priori knowledge to quantify short echo in vivo ${ }^{1} \mathrm{H} \mathrm{MR}$ spectra. Magn Reson Med 34: 17-24.

Stanley JA, Panchalingam K, Keshavan MS, Soares JC, Pettegrew JW (2002b). A post-processing method to accurately quantify $n$ acetylaspartate in short echo time in vivo ${ }^{1} \mathrm{H}$ spectra Proc Int Soc Magn Reson Med (Berkeley CA), 2514.
Stanley JA, Pettegrew JW, Keshavan MS (2000). Magnetic resonance spectroscopy in schizophrenia: methodological issues and findings-part I. Biol Psychiatry 48: 357-368.

Winsberg ME, Sachs N, Tate DL, Adalsteinsson E, Spielman D, Ketter TA (2000). Decreased dorsolateral prefrontal $\mathrm{N}$-acetyl aspartate in bipolar disorder. Biol Psychiatry 47: 475-481.

Yuh WTC, Tali ET, Afifi AK, Sahinoglu K, Gai F, Bergman RA (1994). MRI of Head \& Neck Anatomy. Churchill Livingstone: New York.

Zanardi R, Racagni G, Smeraldi E, Perez J (1997). Differential effects of lithium on platelet protein phosphorylation in bipolar patients and healthy subjects. Psychopharmacology 129: 44-47. 